\title{
Definition and significance of average temperatures in time-dependent solar chromosphere models
}

\author{
W. Rammacher ${ }^{1}$ and M. Cuntz ${ }^{2,3}$ \\ 1 Kiepenheuer-Institut für Sonnenphysik, Schöneckstr. 6, 79104 Freiburg, Germany \\ e-mail: wrammacher@kis.uni-freiburg.de \\ 2 Department of Physics, Science Hall, University of Texas at Arlington (UTA), Arlington, TX 76019-0059, USA \\ e-mail: cuntz@uta.edu \\ 3 Institut für Theoretische Astrophysik, Universität Heidelberg, Albert Überle Str. 2, 69120 Heidelberg, Germany
}

Received 19 October 2004 / Accepted 22 March 2005

\begin{abstract}
We assess different types of average temperatures in time-dependent solar chromosphere models. They include the conventional definition of mean and median temperature, and a formal definition related to the model-dependent hydrogen ionization degree, referred to as ionization temperature. It is found that the latter is always higher than the mean and median temperatures, except in the photosphere, and that the mean temperatures are always higher than the median temperatures, especially in models with frequency spectra. The most dramatic differences are attained in the topmost portion of one of our models with the ionization temperatures up to a factor 150 higher than the mean and median temperatures. The differences between the mean, median, and ionization temperatures are a direct consequence of nonlinearities ("spikyness") of the temperatures in the models mostly due to strong shocks. The main results hold for both acoustic and magnetic models despite significant differences in the initial wave energy fluxes, densities, and geometrical settings.
\end{abstract}

Key words. hydrodynamics - methods: numerical - methods: statistical - magnetohydrodynamics (MHD) Sun: chromosphere - waves

\section{Introduction}

A key element in the description of solar and stellar chromospheres is the assessment and interpretation of timeaveraged temperatures, which are typically given through the analysis of spectral information or evaluation of detailed chromospheric heating simulations (e.g., Schrijver \& Zwaan 2000). Theoretical models of solar and stellar chromospheres have been obtained based on acoustic waves and/or different types of magnetic waves as discussed in recent reviews by Ulmschneider \& Musielak (2003), Roberts (2004), and Musielak (2004). These models take into account the generation, propagation, and dissipation of the waves, as well as the description of emergent emission. Recent models for the Sun have been given by Rammacher \& Ulmschneider (2003), Bogdan et al. (2003), and Wedemeyer et al. (2004). Simulations of chromospheric heating for stars other than the Sun by our group include models by Cuntz et al. (1999), Fawzy et al. (2002), and Rammacher \& Cuntz (2003).

A common feature of these models is the strong temperature fluctuation with time at different atmospheric heights. These fluctuations are due to shock heating and are particularly pronounced if the ionization of hydrogen is described in a self-consistent, time-dependent manner (e.g., Carlsson \& Stein 1994, 1995; Rammacher \& Ulmschneider 2003; Rammacher $\&$ Cuntz 2005), as in this case very high temperature jumps occur immediately behind strong shocks. Other types of models used to derive time-averaged temperatures in the solar chromosphere are semi-empirical models, which interpret spectral information ranging from UV to the optical and infrared regimes. Early models by Vernazza et al. (1981), Maltby et al. (1986), Fontenla et al. (1993), as well as more recent models, assume a smooth increase in temperature as function of height, which is now considered highly unrealistic.

In order to illustrate some of the underlying aspects, we pursue a quantitative analysis of different types of timeaveraged temperatures taking up-to-date theoretical solar chromosphere models as examples. The different types of average temperatures include the mean temperature $T_{\text {mean }}$, the median temperature $T_{\text {median }}$, and the ionization temperature $T_{\text {ion }}$ inferred from the hydrogen ionization degree. Our paper is structured as follows. In Sect. 2, we describe the basics of the (magneto-)hydrodynamic code and provide different definitions of average temperature. In Sect. 3, we give our results and discussion. The summary and conclusions are given in Sect. 4 .

\section{Methods}

\section{1. (Magneto-)hydrodynamic computer code}

The solar chromospheric models based on acoustic waves or longitudinal flux-tube waves are calculated using the 
numerical code by Rammacher \& Ulmschneider (2003) and Cuntz \& Rammacher (2005). This code allows one to compute one-dimensional time-dependent wave propagation in solar and stellar atmospheres, while incorporating the treatment of hydrogen ionization through solving the time-dependent statistical rate equations (see also Carlsson \& Stein 1992, 1995, for another version of that method). The solutions of the (magneto-)hydrodynamic equations are obtained together with the radiative transfer equation considering departures from local thermodynamic equilibrium (NLTE). Furthermore, the code also considers ionization of magnesium and calcium. The thermodynamical relationships, as well as the Rankine-Hugoniot relations across the shocks, are solved in a self-consistent timedependent manner. The inner boundary condition allows the treatment of upward propagating waves, whereas the outer boundary condition allows the waves to be transmitted.

The code is suitable for the treatment of monochromatic waves and frequency spectra. With respect to monochromatic wave models, the dynamic structure of the acoustic and magnetic chromosphere models is found to be given by a timedependent balance among shock heating, radiative and hydrodynamic cooling, and ionization. As expected, the ionization balance of $\mathrm{H}^{+} / \mathrm{H}, \mathrm{Ca} \mathrm{II} / \mathrm{Ca}$, and $\mathrm{Mg} \mathrm{II} / \mathrm{Mg}$ is essentially unaffected by the localized energy dissipation behind the shocks as already found in previous models for the Sun (Rammacher \& Ulmschneider 2003). This allows the post-shock temperatures to increase beyond the values typically attained in models with time-independent (i.e., instantaneous) ionization. In fact, the formation of $\mathrm{Ca}$ II and $\mathrm{Mg}$ II emission does not occur immediately behind the shocks and is thus considerably reduced compared to previous models.

\subsection{Definition of average temperatures}

The concept of average temperatures is highly problematic in shock-dominated atmospheres. In empirical models, temperatures are typically derived from other quantities such as element ionization stages and electron densities. These quantities are, however, not unambiguously dependent on temperature, thus yielding different types of average temperatures. An observed electron density, for instance, can correspond to radically different temperatures depending on the adopted chromospheric model.

In the following, we introduce three different types of average temperatures to demonstrate the effect of ambiguity. In the existing literature, average quantities are frequently defined at given mass column densities, which is highly appropriate for those observed spectral diagnostics that tend to be formed at a given optical depth. In the present paper, time averages are calculated at a given geometric height (Eulerian frame).

The first type is the "classical" arithmetic mean temperature, defined as $T_{\text {mean }}=\sum_{i=1}^{N} \delta_{i} T_{i}$. Here $\delta_{i}$ is given by the weights of the $N$ contributions of $T_{i}$. In case of nonequidistant time-spacing, the values of $\delta_{i}$ will be different, which may occur both in theoretical and observational studies. In theoretical models, $\delta_{i}$ is often chosen differently to resolve temperature jumps associated with the spiky structure of the atmosphere.
The second type of average temperature is the median temperature $T_{\text {median }}$, which during the timespan of averaging is defined as temperature where half of the time the instant temperature is higher and half of the time the instant temperature is lower than that temperature. The difference between $T_{\text {mean }}$ and $T_{\text {median }}$ is a measure of the skewness of the time-dependent temperature fluctuation at a given height as, e.g., introduced by strong shocks.

The third type of average temperature can be obtained from the reanalysis of the (model-dependent) time-averaged hydrogen ionization degree. In principle, we find that

$\frac{n_{\mathrm{c}} n_{\mathrm{e}}}{n_{1}}=f(T)$,

which is valid for a one-level plus continuum atom in LTE. Here $n_{1}, n_{\mathrm{c}}$, and $n_{\mathrm{e}}$ represent the number densities of the first and continuum level of hydrogen, and $n_{\mathrm{e}}$ is the electron density. Equation (1) thus allows us to obtain a new type of time-averaged temperature given by the mean values of $n_{1}, n_{\mathrm{c}}$, and $n_{\mathrm{e}}$. For a two-level plus continuum atom in time-dependent NLTE approximation, a slightly more complicated approach is required. In this case, we find

$$
\begin{aligned}
& \frac{n_{1}}{n_{\mathrm{c}}}=b_{1} \chi_{\mathrm{c} 1}^{-1} \\
& \frac{n_{2}}{n_{\mathrm{c}}}=b_{2} \chi_{\mathrm{c} 2}^{-1} \\
& n_{\mathrm{c}}=n_{\mathrm{H}}-n_{1}-n_{2}
\end{aligned}
$$

with $n_{1}, n_{2}$, and $n_{\mathrm{c}}$ as NLTE number densities of the first, second, and continuum level of hydrogen, and $n_{\mathrm{H}}$ as total hydrogen number density. Moreover, $\chi_{\mathrm{c} 1}$ and $\chi_{\mathrm{c} 2}$ indicate the Saha factors for the first and second hydrogen ionization level, respectively.

The departure coefficients $b_{1}$ and $b_{2}$ for the first and second level, normalized to the continuum, are

$$
\begin{aligned}
& b_{1}=\frac{n_{1} n_{\mathrm{c}}^{\star}}{n_{1}^{\star} n_{\mathrm{c}}} \\
& b_{2}=\frac{n_{2} n_{\mathrm{c}}^{\star}}{n_{2}^{\star} n_{\mathrm{c}}}
\end{aligned}
$$

with $n_{1}^{\star}$ and $n_{2}^{\star}$ as LTE quantities. We also obtain

$$
\begin{aligned}
& \chi_{\mathrm{c} 1}^{-1}=\frac{n_{1}^{\star}}{n_{\mathrm{c}}^{\star}}=n_{\mathrm{e}}\left(\frac{h^{2}}{2 \pi m_{\mathrm{e}} k T}\right)^{3 / 2} \mathrm{e}^{E_{1} / k T} \\
& \chi_{\mathrm{c} 2}^{-1}=\frac{n_{2}^{\star}}{n_{\mathrm{c}}^{\star}}=4 n_{\mathrm{e}}\left(\frac{h^{2}}{2 \pi m_{\mathrm{e}} k T}\right)^{3 / 2} \mathrm{e}^{E_{2} / k T}
\end{aligned}
$$

with $E_{1}$ and $E_{2}$ given as $E_{1}=E_{\mathrm{H}}$ and $E_{2}=\frac{1}{4} E_{\mathrm{H}}, E_{\mathrm{H}}=$ $13.595 \mathrm{eV}$ as ionization energy of the hydrogen ground state, and all other symbols having their usual meaning.

Due to the nonlinearities and the time-dependent behavior of the relevant variables (see Fig. 1), Eqs. (2) to (8) cannot hold simultaneously if $n_{\mathrm{c}} / n_{\mathrm{H}}, n_{\mathrm{e}}, b_{1}, b_{2}$, and $T$ are represented by their mean values. On the other hand, if mean values are selected for those variables except $T$, Eqs. (2) to (8) can be utilized to recalculate $T$, which shall be defined as "ionization temperature" $T_{\text {ion }}$, a new type of "average temperature". 


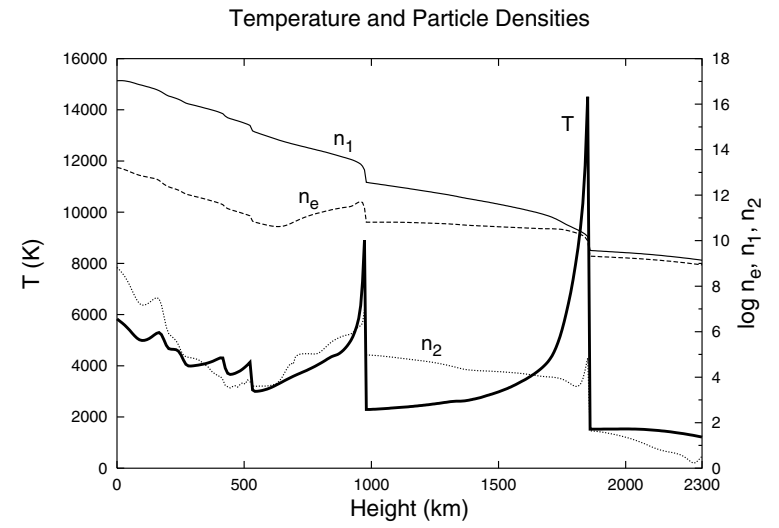

Fig. 1. Time-step of the model representing stochastic longitudinal flux-tube waves after $2366 \mathrm{~s}$. Shown are temperature $T$ (thick-solid) and particle densities $n_{1}$ (thin-solid), $n_{2}$ (dashed), and $n_{\mathrm{e}}$ (dotted line).

The fact that $T_{\text {ion }}$ deviates from $T_{\text {mean }}$, as well as from $T_{\text {median }}$ in most cases, is a direct consequence of the behavior of the various particle densities, as well as $b_{1}$ and $b_{2}$. This is particularly evident in models with strong shocks and noninstantaneous ionization.

Note that $T_{\text {ion }}$ is largely affected by the time-averaged electron number density, which is chiefly determined by the increased ionization degree in the post-shock regions, although there are significant modifications of this picture due to noninstantaneous ionization. This will result in $T_{\text {ion }}$ being higher than $T_{\text {mean }}$ and $T_{\text {median. }}$. Nonetheless, the above given definition of $T_{\text {ion }}$ is only one of several possibilities. Theoretically, for any ionized stage of an element (e.g., Ca II, Mg II) such a ionization temperature could be defined. Undisputably, however, hydrogen is by far the most abundant element in stellar atmospheres, and furthermore ionization of hydrogen is by far the most important process for determining the electron density particularly in the upper solar chromosphere, making the above choice of $T_{\text {ion }}$ most appropriate.

In empirical models, a further type of "average temperature" arises that is not directly related to any of the temperatures discussed here. This temperature is defined as reproducing quantities as close as possible to the observed temporal means. How this semi-empirical temperature relates to any physical temperature in a dynamic model is not clear and will depend on the observable(s) used in the semi-empirical model. If the observable is the mean intensity in the UV (as is the case in VAL3C), the semi-empirical temperature will always sample the higher temperatures exhibited in the dynamic model rather than temperatures close to $T_{\text {mean }}$ or $T_{\text {median }}$.

\section{Results and discussion}

\section{1. (Magneto-)hydrodynamic model computations}

In the following, we consider three different models: a monochromatic acoustic model (model 1), a monochromatic longitudinal flux-tube model (model 2), and a stochastic longitudinal flux-tube model (model 3). The construction of these models requires the computation of input parameters. They include wave energy fluxes for each of those models, the wave frequency spectrum for the stochastic model, and the representative wave period in the monochromatic models.

The computation of the acoustic energy flux follows the work by Ulmschneider et al. (1996), who incorporated an improved description of the spatial and temporal spectrum of the turbulent flow. These models utilize an extended Kolmogorov spectrum with a modified Gaussian frequency factor. The acoustic energy flux is found to be dependent on the mixinglength parameter $\alpha$, for which we use $\alpha=2$ as indicated by solar studies (Steffen 1993; Robinson et al. 2003). Similar investigations in the case of longitudinal flux-tube waves have been presented by Ulmschneider \& Musielak (1998). The initial wave energy flux for our acoustic model is thus given as $F_{\mathrm{M}}=1.7 \times 10^{8} \mathrm{erg} \mathrm{cm}^{-2} \mathrm{~s}^{-1}$, whereas the initial wave energy flux for the longitudinal flux-tube models is given as $F_{\mathrm{M}}=5.6 \times 10^{8} \mathrm{erg} \mathrm{cm}^{-2} \mathrm{~s}^{-1}$. The work by Ulmschneider \& Musielak (1998) also provides information on the frequency spectrum for polychromatic longitudinal waves. The wave period at the maximum of the energy distribution for both the acoustic and longitudinal frequency spectrum is found to be close to $60 \mathrm{~s}$. Therefore, this value will be used for the two monochromatic wave computations in this study.

Furthermore, we must also define the geometrical setting. Model 1 is based on plane-parallel geometry, while for model 2 and model 3 flux-tube geometry is assumed. The flux-tube geometry is based on the idea that at very low atmospheric heights, unobstructed spreading occurs, whereas at larger heights, the influence of neighboring flux tubes (e.g., Frutiger \& Solanki 2001; Schrijver \& Title 2003) dominates, thus leading to reduced spreading. Eventually, a distinct tube opening radius is reached (see Rammacher \& Ulmschneider 1989; Fawzy et al. 1998, 2002; Rammacher \& Ulmschneider 2003 , for earlier work). For the flux-tube model in this paper, the bottom opening radius is set as $55 \mathrm{~km}$, corresponding to half of the photospheric density scale-height. The top opening radius of the flux tube is assumed to be $175 \mathrm{~km}$. Therefore, the top opening area of the tube is about ten times larger than its bottom area. At relatively low heights, exponential spreading occurs. Full spreading is attained at a height of $620 \mathrm{~km}$.

Temperature contour maps of the monochromatic and stochastic longitudinal wave models based on the data used for the calculation of the different types of time-averaged temperatures are given in Fig. 2.

\subsection{Comparison of average temperatures}

In the following, we discuss results for the different types of average temperatures obtained for our acoustic and magnetic wave models. In that respect, we focus on timespans where the switch-on phase of the wave entering the undisturbed atmosphere has completely subsided. In the case of the monochromatic acoustic and magnetic wave model (models 1 and 2, respectively), this status has been achieved after 50 wave periods entered the atmospheric computational domain. Note that due to the inclusion of time-dependent ionization, this timespan is longer than in previous models with time-independent 


\section{Temperature Distribution}
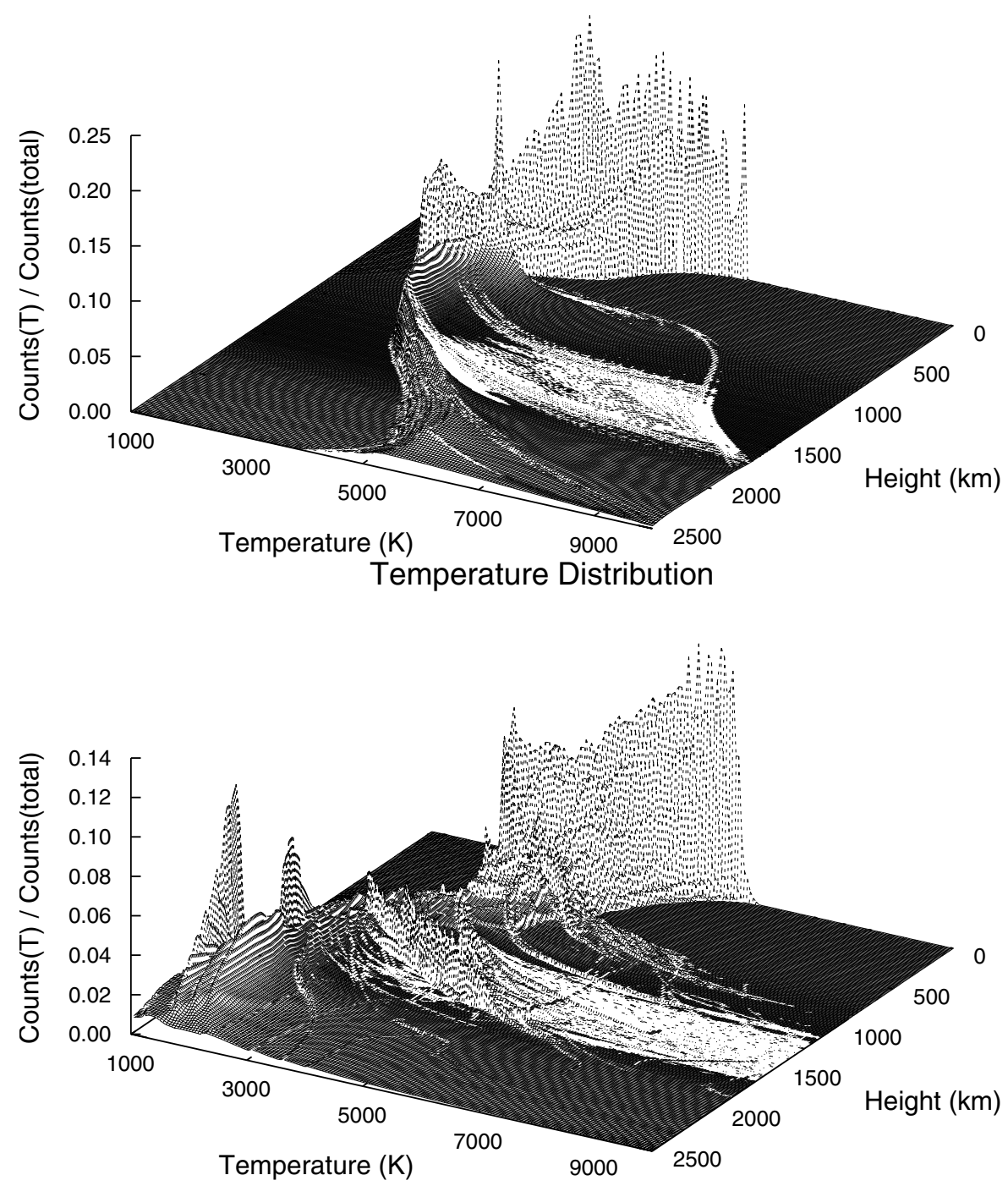

Fig. 2. Temperature contour maps for the monochromatic longitudinal wave model (model 2) (top) and stochastic longitudinal wave model (model 3) (bottom) based on the data also taken for the calculation of the different types of time-averaged temperatures.

ionization where dynamic equilibrium was typically reached after 20 wave periods.

Figure 3 shows the behavior of the pre-shock and postshock temperatures of model $1, T_{1}$ and $T_{2}$, respectively. Evidently, only very small variations of $T_{1}$ and $T_{2}$ exist unequivocal evidence of dynamical equilibrium. In the case of the stochastic longitudinal wave model (model 3), no dynamic equilibrium persists. Nevertheless, time-averaged temperatures were calculated after a sufficiently long elapsed time to ensure that the dynamics of the wave is determined by the employed frequency spectrum rather than switch-on effects. The timespans for the calculation of time-averaged temperatures for models 1-3 have been $1021 \mathrm{~s}, 1861 \mathrm{~s}$, and $909 \mathrm{~s}$, respectively.

Figure 4 shows the behavior of $T_{\text {mean }}, T_{\text {median }}$, and $T_{\text {ion }}$ for models $1-3$. It is found that $T_{\text {ion }}$ is higher than both $T_{\text {mean }}$ and $T_{\text {median }}$, and $T_{\text {mean }}$ is higher than $T_{\text {median }}$ at all heights except in the photosphere. For model $1, T_{\text {median }}, T_{\text {mean }}$, and $T_{\text {ion }}$ at $1000 \mathrm{~km}$ are $3810 \mathrm{~K}, 4140 \mathrm{~K}$, and $6100 \mathrm{~K}$; they are $3790 \mathrm{~K}$, $4270 \mathrm{~K}$, and $6690 \mathrm{~K}$ at $1500 \mathrm{~km}$, and $5310 \mathrm{~K}, 5840 \mathrm{~K}$, and
$8050 \mathrm{~K}$ at $2000 \mathrm{~km}$, respectively. In model 2, those temperatures are generally higher, but it is found that the differences between the different types of average temperatures at a given height are about the same, irrespective of the different initial wave energy fluxes, densities, and geometries of the two models. This phenomenon is an intimate consequence of the limiting shock strength behavior of those waves as previously discussed by Ulmschneider (1970), Cuntz \& Ulmschneider (1988), and Cuntz (2004). For further results on the propagation of longitudinal tube waves with the consideration of timedependent ionization see Rammacher \& Cuntz (2005).

A drastically different picture concerning the different types of average temperatures emerges for model 3 . Here it is found that $T_{\text {median }}, T_{\text {mean }}$, and $T_{\text {ion }}$ at $1000 \mathrm{~km}$ are given as $3250 \mathrm{~K}$, $3450 \mathrm{~K}$, and $7000 \mathrm{~K}$; at $1500 \mathrm{~km}$, they are given as $2440 \mathrm{~K}$, $3330 \mathrm{~K}$, and $8650 \mathrm{~K}$; and at $2000 \mathrm{~km}$, they are given as $1835 \mathrm{~K}$, $2890 \mathrm{~K}$, and $19200 \mathrm{~K}$, respectively. These extremely large differences between $T_{\text {mean }}$ and $T_{\text {median }}$, on one hand, and $T_{\text {ion }}$, on the other hand, particularly at large chromospheric heights, are 
Table 1. Time-averaged $b_{1}$ and $b_{2}$ values.

\begin{tabular}{cccccccc}
\hline \hline Model & \multicolumn{2}{c}{ Model 1 } & \multicolumn{3}{c}{ Model 2 } & \multicolumn{2}{c}{ Model 3 } \\
\cline { 2 - 9 } & $\begin{array}{c}\text { Height } \\
(\mathrm{km})\end{array}$ & $b_{1}$ & $b_{2}$ & $b_{1}$ & $b_{2}$ & $b_{1}$ & $b_{2}$ \\
& $\ldots$ & $\ldots$ & $\ldots$ & $\ldots$ & $\ldots$ & $\ldots$ \\
\hline 500 & $4.43 \mathrm{E}-01$ & $3.12 \mathrm{E}-01$ & $3.82 \mathrm{E}-02$ & $8.99 \mathrm{E}-03$ & $4.21 \mathrm{E}-02$ & $6.63 \mathrm{E}-02$ \\
1000 & $5.27 \mathrm{E}+00$ & $1.73 \mathrm{E}+00$ & $6.73 \mathrm{E}+00$ & $1.50 \mathrm{E}+00$ & $1.01 \mathrm{E}+02$ & $1.34 \mathrm{E}+01$ \\
1500 & $1.05 \mathrm{E}+01$ & $1.89 \mathrm{E}+00$ & $4.18 \mathrm{E}+01$ & $3.71 \mathrm{E}+00$ & $4.61 \mathrm{E}+03$ & $7.38 \mathrm{E}+01$ \\
2000 & $8.02 \mathrm{E}+02$ & $1.27 \mathrm{E}+01$ & $8.31 \mathrm{E}+03$ & $3.75 \mathrm{E}+01$ & $1.25 \mathrm{E}+08$ & $1.35 \mathrm{E}+04$ \\
2400 & $5.44 \mathrm{E}+03$ & $1.69 \mathrm{E}+01$ & $4.37 \mathrm{E}+04$ & $4.66 \mathrm{E}+01$ & $6.13 \mathrm{E}+12$ & $1.26 \mathrm{E}+07$ \\
\hline
\end{tabular}

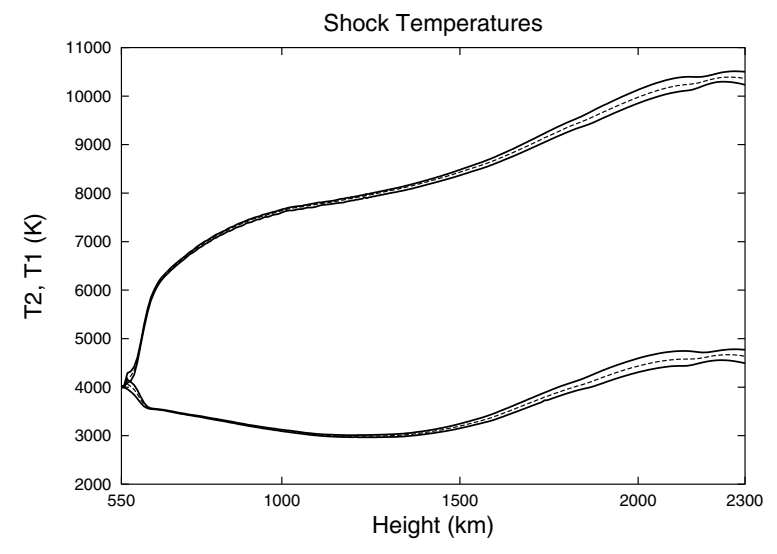

Fig. 3. Minimum and maximum temperature values for the pre-shock and post-shock temperatures $T_{1}$ and $T_{2}$ (solid lines) given by the monochromatic acoustic wave model (model 1) after dynamic equilibrium has been attained. The average values are given as dotted lines. Note that shock formation occurs near $570 \mathrm{~km}$.

due to the highly nonlinear behavior of the temperature that is a consequence of the occurrence of strong shocks formed by merging of shocks in the stochastic wave field.

Very low temperatures are attained for $T_{\text {median }}$, especially at large chromospheric heights, a consequence of the presence of strong shocks, which noticeably affect $T_{\text {mean }}$, but barely $T_{\text {median }}$. In model 3, $T_{\text {median }}$ ranges between 1600 and $1850 \mathrm{~K}$ at heights beyond $2000 \mathrm{~km}$, thus constituting absurdly low values for any type of chromospheric structure. On the other hand, $T_{\text {ion }}$ is found to reach $254000 \mathrm{~K}$ at $2480 \mathrm{~km}$, the outermost point of our model, thus becoming a factor of more than 150 higher than $T_{\text {median. }}$. An important property affecting $T_{\text {ion }}$ is the extreme NLTE effect impacting the degree of hydrogen ionization (see Table 1); e.g., at $2000 \mathrm{~km}$ in model $3, b_{1}$ and $b_{2}$ are identified as $1.25 \times 10^{8}$ and $1.35 \times 10^{4}$, respectively. The fact that in the stochastic magnetic wave model, contrary to the monochromatic magnetic wave model, very low temperatures are reached at large heights most of the time is also visible from the temperature contour maps (Fig. 2).

The uppermost portion of the chromosphere heated by stochastic longitudinal flux-tube waves is the most drastic case of differences in average temperatures, according to the adopted definitions. A cool chromosphere, identified by its mean temperature, can thus show the same mean degree of hydrogen ionization as a "classical" chromosphere with a relatively high mean temperature. The customary definition of mean and median temperature therefore seems inappropriate for describing shock-heated atmospheres - assuming that such strong shocks as in model 3 (see Fig. 1) exist in reality. This

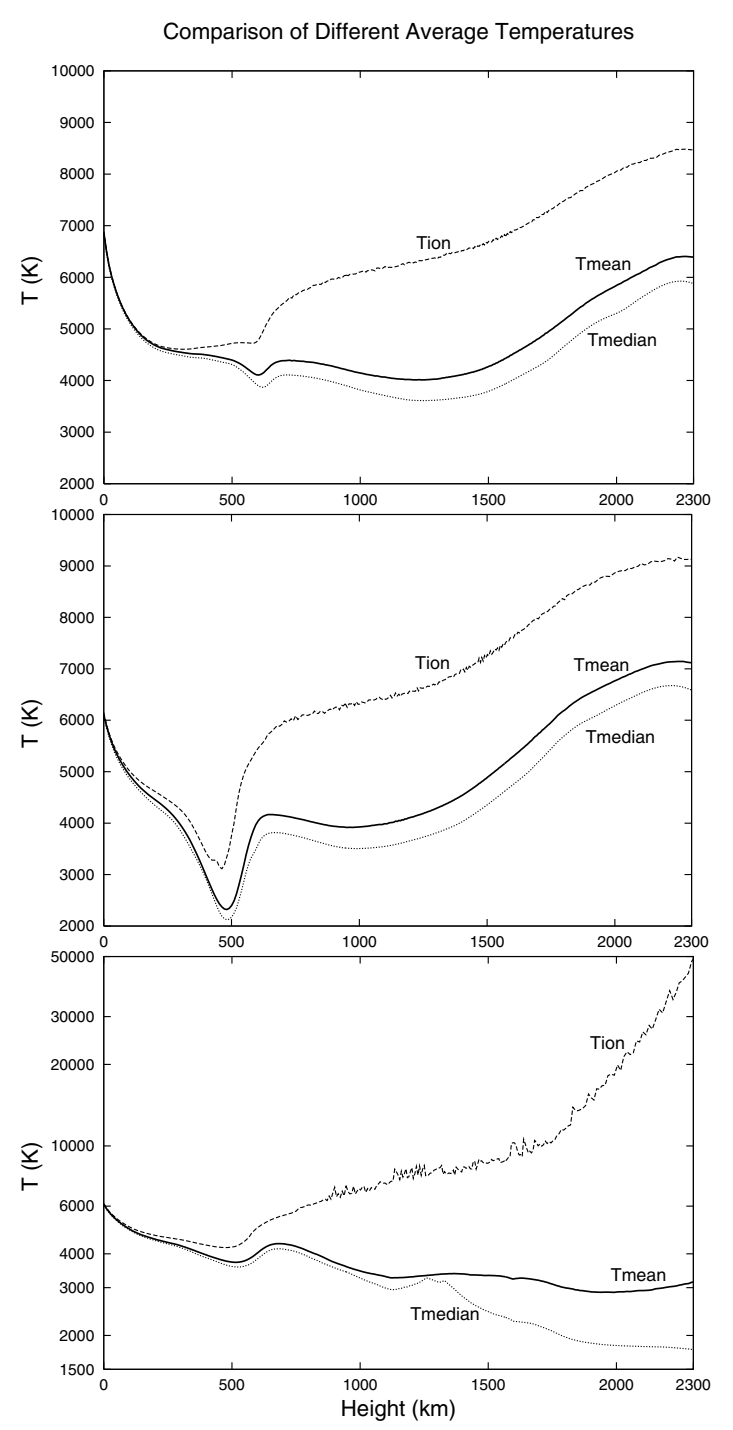

Fig. 4. Comparison of $T_{\text {ion }}, T_{\text {mean }}$, and $T_{\text {median }}$ for the monochromatic acoustic wave model (model 1) (top), the monochromatic longitudinal wave model (model 2) (middle), and the stochastic longitudinal wave model (model 3) (bottom). Note that the $T$-scale of model 3 is increased by a factor of 5 and is given in logarithmic units.

pivotal issue is part of an ongoing debate that will be briefly visited in the next section.

\section{Summary and conclusions}

We have discussed the significance of different types of average temperatures with respect to time-dependent solar chromosphere models. Specifically, we considered a monochromatic acoustic model, a monochromatic longitudinal flux-tube 
model, and a stochastic longitudinal flux-tube model. The different definitions of average temperature include its conventional definition ( $\left.T_{\text {mean }}\right)$, the median of the time-dependent temperature $\left(T_{\text {median }}\right)$, and a formal definition given by the analysis of the time-averaged hydrogen ionization degree $\left(T_{\text {ion }}\right)$.

For all three models, we found that beyond photospheric levels, $T_{\text {mean }}$ is always higher than $T_{\text {median }}$, and $T_{\text {ion }}$ is always higher than $T_{\text {mean }}$. In the stochastic wave model, the differences between the three different average temperatures are found to increase with height. This result is a direct consequence of the nonlinearities and time fluctuations exhibited by the wave simulations. For instance, in the stochastic wave model $T_{\text {ion }}$ is found to exceed $40000 \mathrm{~K}$ beyond $2200 \mathrm{~km}$, whereas $T_{\text {mean }}$ is found to stay below $4500 \mathrm{~K}$ at all heights above the photosphere. On the other hand, $T_{\text {median }}$ is as low as $1600 \mathrm{~K}$ as reached at heights above $2000 \mathrm{~km}$ in the stochastic longitudinal flux-tube model. It is also found that in monochromatic wave simulations, $T_{\text {mean }}$ gradually increases with height, as previously noted by Rammacher \& Ulmschneider (2003); whereas in the stochastic wave simulation, $T_{\text {mean }}$ decreases with height, consistent with earlier results by Carlsson \& Stein $(1994,1995)$ and Rammacher \& Ulmschneider (2003).

An important question closely related to the topic of this paper concerns the realistic behavior of the "real" time-averaged temperature $T_{\text {mean }}$ exhibited by the magnetic and nonmagnetic solar chromosphere for the different atmospheric heights. This is the focus of an ongoing debate with a wide variety of views. On one hand, it has been argued that $T_{\text {mean }}$ increases with height to account for the variety of UV diagnostics (e.g., Kalkofen et al. 1999). On the other hand, Carlsson \& Stein (1994, 1995 ) originally proposed that $T_{\text {mean }}$ decreases with height in stark contrast to "classical" solar chromosphere models. In fact, Ayres (2002) argued that this type of model is "not even cold enough", as suggested by the off-limb solar CO emission. An intermediate possibility is given by the concept of thermal bifurcation and radiative instability, which points to high and low temperatures occurring at the same mass column density within the outer solar atmosphere (e.g., Ayres 1981; Muchmore \& Ulmschneider 1985; Solanki et al. 1994; Cuntz \& Muchmore 1994, and others).

A different type of approach consists in considering effects due to 3-D propagation of the waves as discussed by Wedemeyer et al. (2004), Kalkofen (2004), and Ulmschneider et al. (2005), among others. Ulmschneider et al. (2005) argue that acoustic waves are expected to propagate in cone-shaped channels, thus avoiding significant shock overtaking and the formation of strong shocks (as obtained in model 3), but generating a weak quasi-steady level of background heating. If correct, acoustic and magnetic waves may therefore be able to produce a quasi-monotonic increase in temperature with height, consistent with previous semi-empirical models.

Acknowledgements. This work has been supported by the German Research Foundation DFG through grant UL57/33-1 (W. R.) and by NSF through grant ATM-0087184 (M. C.). We also appreciate comments by an anonymous referee on the temperature definition in empirical chromosphere models.

\section{References}

Ayres, T. R. 1981, ApJ, 244, 1064

Ayres, T. R. 2002, ApJ, 575, 1104

Bogdan, T. J., Carlsson, M., Hansteen, V., et al. 2003, ApJ, 599, 626

Carlsson, M., \& Stein, R. F. 1992, ApJ, 397, L59

Carlsson, M., \& Stein, R. F. 1994, in Chromospheric Dynamics, ed.

M. Carlsson (Oslo: Institute of Theoretical Astrophysics), 47

Carlsson, M., \& Stein, R. F. 1995, ApJ, 440, L29

Cuntz, M. 2004, A\&A, 420, 699

Cuntz, M., \& Muchmore, D. O. 1994, ApJ, 433, 303

Cuntz, M., \& Rammacher, W. 2005, ApJ, submitted

Cuntz, M., \& Ulmschneider, P. 1988, A\&A, 193, 119

Cuntz, M., Rammacher, W., Ulmschneider, P., Musielak, Z. E., \& Saar, S. H. 1999, ApJ, 522, 1053

Fawzy, D. E., Ulmschneider, P., \& Cuntz, M. 1998, A\&A, 336, 1029

Fawzy, D. E., Rammacher, W., Ulmschneider, P., Musielak, Z. E., \& Stępień, K. 2002, A\&A, 386, 971

Fontenla, J. M., Avrett, E. H., \& Loeser, R. 1993, ApJ, 406, 319

Frutiger, C., \& Solanki, S. K. 2001, A\&A, 369, 646

Kalkofen, W., Ulmschneider, P., \& Avrett, E. H. 1999, ApJ, 521, L141

Kalkofen, W. 2004, in Stars as Suns: Activity, Evolution and Planets, ed. A. K. Dupree \& A. O. Benz (San Francisco: ASP), IAU Symp., 219, 115

Maltby, P., Avrett, E. H., Carlsson, M., et al. 1986, ApJ, 306, 284

Mihalas, D. 1978, Stellar Atmospheres (San Francisco: W. H. Freeman and Co.)

Muchmore, D., \& Ulmschneider, P. 1985, A\&A, 142, 393

Musielak, Z. E., Rosner, R., Stein, R. F., \& Ulmschneider, P. 1994, ApJ, 423, 474

Musielak, Z. E. 2004, in Stars as Suns: Activity, Evolution and Planets, ed. A. K. Dupree \& A. O. Benz (San Francisco: ASP), IAU Symp., 219, 437

Rammacher, W., \& Cuntz, M. 2003, ApJ, 594, L51

Rammacher, W., \& Cuntz, M. 2005, in Cool Stars, Stellar Systems, and the Sun, ed. F. Favata et al., ESA SP, in press

Rammacher, W., \& Ulmschneider, P. 1989, in Solar and Stellar Granulation, ed. R. J. Rutten \& G. Severino (Dordrecht: Kluwer), 589

Rammacher, W., \& Ulmschneider, P. 2003, ApJ, 589, 988

Roberts, B. 2004, in SOHO 13: Waves, Oscillations and Small Scale Transient Events in the Solar Atmosphere: A Joint View of Soho and TRACE, ESA SP-547, in press

Robinson, F. J., Demarque, P., Li, L. H., et al. 2003, MNRAS, 340, 923

Schrijver, C. J., \& Title, A. M. 2003, ApJ, 597, L165

Schrijver, C. J., \& Zwaan, C. 2000, Solar and Stellar Magnetic Activity (Cambridge: Cambridge University Press)

Solanki, S. K., Livingston, W., \& Ayres, T. 1994, Science, 263, 64

Steffen, M. 1993, Habilitation Thesis, Univ. Kiel, Germany

Ulmschneider, P. 1970, Sol. Phys., 12, 403

Ulmschneider, P., \& Musielak, Z. E. 2003, in Current Theoretical Models and Future High Resolution Solar Observations: Preparing for ATST, ed. A. A. Pevtsov \& H. Uitenbroek (San Francisco: ASP Conf. Ser.), 286, 363

Ulmschneider, P., \& Musielak, Z. E. 1998, A\&A, 338, 311

Ulmschneider, P., Rammacher, W., Musielak, Z. E., \& Kalkofen, W. 2005, ApJ, submitted

Ulmschneider, P., Theurer, J., \& Musielak, Z. E. 1996, A\&A, 315, 212

Vernazza, J. E., Avrett, E. H., \& Loeser, R. 1981, ApJS, 45, 635

Wedemeyer, S., Freytag, B., Steffen, M., Ludwig, H.-G., \& Holweger, H. 2004, A\&A, 414, 1121 